\title{
Hausdorff measure of nodal sets of analytic Steklov eigenfunctions
}

\author{
Steve ZeLditch
}

Let $(\Omega, g)$ be a real analytic Riemannian manifold with real analytic boundary $\partial \Omega$. Let $\psi_{\lambda}$ be an eigenfunction of the Dirichlet-toNeumann operator $\Lambda$ of $(\Omega, g, \partial \Omega)$ of eigenvalue $\lambda$. Let $\mathcal{N}_{\lambda}$ be its nodal set. Then, there exists a constant $C>0$ depending only on $(\Omega, g, \partial \Omega)$ so that

$$
\mathcal{H}^{n-2}\left(\mathcal{N}_{\lambda}\right) \leq C \lambda
$$

This proves a conjecture of F. H. Lin and K. Bellova.

This article is concerned with the Hausdorff $\mathcal{H}^{n-2}$ measure of the nodal sets

$$
\mathcal{N}_{\lambda}=\left\{x \in \partial \Omega: \psi_{\lambda}(x)=0\right\} \subset \partial \Omega
$$

of Steklov eigenfunctions of eigenvalue $\lambda$ of a domain $\Omega \subset \mathbb{R}^{n}$ in the real analytic case. The Steklov eigenvalue problem on a domain $\Omega$ is,

$$
\left\{\begin{array}{l}
\Delta u(x)=0, \quad x \in \Omega, \\
\frac{\partial u}{\partial \nu}(x)=\lambda u(x), \quad x \in \partial \Omega .
\end{array}\right.
$$

It is often assumed that $\Omega \subset \mathbb{R}^{n}$ is a bounded $C^{2}$ domain with Euclidean metric, but the problem may be posed on a bounded domain in any Riemannian manifold. The eigenvalue problem may be reduced to the boundary, and $\psi_{\lambda}$ is an eigenfunction

$$
\Lambda \psi_{\lambda}=\lambda \psi_{\lambda}
$$

of the Dirichlet-to-Neumann operator

$$
\Lambda f=\left.\frac{\partial u}{\partial \nu}(x)\right|_{\partial \Omega} .
$$

Research partially supported by NSF grant DMS-1206527. 
Here, $u$ is the harmonic extension of $f$,

$$
\left\{\begin{array}{l}
\Delta u(x)=0, \quad x \in \Omega, \\
u(x)=f(x), \quad x \in \partial \Omega .
\end{array}\right.
$$

$\Lambda$ is a positive elliptic self-adjoint pseudo-differential operator of order one on $L^{2}(\partial \Omega, d S)$ and there exists an orthonormal basis $\left\{\psi_{j}\right\}$ of eigenfunctions

$$
\Lambda \psi_{j}=\lambda_{j} \psi_{j}, \quad \psi_{j} \in C^{\infty}(\partial \Omega), \quad \int_{\partial \Omega} \psi_{j} \psi_{k} d S=\delta_{j k}
$$

where $d S$ is the surface measure. We order the eigenvalues in ascending order $0=\lambda_{0}<\lambda_{1} \leq \lambda_{2} \leq \cdots$, counted with multiplicty (see e.g. [DG]).

In a recent article, Bellova-Lin [BL] proved that

$$
\mathcal{H}^{n-2}\left(\mathcal{N}_{\lambda}\right) \leq C \lambda^{6}
$$

when $\Omega \subset \mathbb{R}^{n}$ is a bounded real analytic Euclidean domain. They suggest that the optimal result is $\mathcal{H}^{n-2}\left(\mathcal{N}_{\lambda}\right) \leq C \lambda$. This is the analogue for the Steklov problem of the upper bound half of Yau's conjecture [Y1, Y2] that

$$
c_{M, g} \lambda \leq \mathcal{H}^{n-1}\left(\mathcal{N}_{\varphi_{\lambda}}\right) \leq C_{M, g} \lambda
$$

for nodal sets of Laplace eigenfunctions of $C^{\infty}$ metrics.

The purpose of this article is to prove this upper bound for bounded real analytic domains in general real analytic Riemannian manifolds.

Theorem 1. Let $(\Omega, g)$ be a compact real analytic Riemannian manifold with real analytic boundary $\partial \Omega$. Let $\psi_{\lambda}$ be an eigenfunction of the Dirichletto-Neumann operator $\Lambda$ of $(\Omega, g, \partial \Omega)$ of eigenvalue $\lambda$, and $\mathcal{N}_{\lambda}$ be its nodal set as above. Then, there exists a constant $C>0$ depending only on $(\Omega, g, \partial \Omega)$ so that

$$
\mathcal{H}^{n-2}\left(\mathcal{N}_{\lambda}\right) \leq C \lambda
$$

It is not hard to find examples of $(\Omega, g, \partial \Omega)$ and $\psi_{\lambda}$ where the upper bound is achieved, for instance on a hemisphere of a round sphere. But it is not clear that it is attained by a sequence of Steklov eigenfunctions on every $(\Omega, g, \partial \Omega)$, or more stringently that it is obtained by every sequence of eigenfunctions. In the setting of real analytic Riemannian manifolds $(M, g)$, it is proved in $[\mathrm{DF}]$ that there exists $C>0$ depending only on the metric $g$ so that $\mathcal{H}^{n-1}\left(\mathcal{N}_{\lambda}\right) \geq C \lambda$ (this is the lower bound half of $\left.(3)\right)$. Since $\operatorname{dim} \partial \Omega=$ 
$n-1$, the analogous lower bound for the real analytic Steklov problem would be $\mathcal{H}^{n-2}\left(\mathcal{N}_{\lambda}\right) \geq C \lambda$, where $C$ depends only on $(\Omega, g, \partial \Omega)$. However the key existence result for $\Delta$-eigenfunctions of eigenvalue $\lambda^{2}$, that every ball of radius $\frac{C}{\lambda}$ contains a zero of $\varphi_{\lambda}$, does not seem to be known for the Steklov problem (1); here and below $\Delta$ is the positive Laplacian. A recent result of Wang-Zhu [WZ] gives a power law lower bound on $\mathcal{H}^{n-2}\left(\mathcal{N}_{\lambda}\right)$ which is consistent with known lower bounds in the $C^{\infty}$ case. It would be interesting to see if the complex analytic methods of this article can improve the lower bounds in the analytic case.

\subsection{Outline of the proof of Theorem 1}

The key to proving the sharp upper bound in the generality of Theorem 1 is to use the wave group

$$
U(t)=e^{i t \Lambda}: L^{2}(\partial \Omega) \rightarrow L^{2}(\partial \Omega)
$$

generated by $\Lambda$. As mentioned above, $\Lambda$ is a positive elliptic self-adjoint pseudo-differential operator of order one, with principal symbol equal to the metric norm $|\xi|_{g_{\partial \Omega}}$ of the boundary metric (see e.g. [T, LU]). Its wave group is therefore a Fourier integral operator by the methods and results of [Hor, DG]. As in [Z] we study nodal sets by analytically continuing the Schwartz kernel of the wave group to imaginary time $t+i \tau$ with $\tau>0$, and to the complexification $(\partial \Omega)_{\mathbb{C}}$ of $\partial \Omega$. The analytic continuation in time and in the first space variable defines the Poisson wave kernel

$$
U_{\mathbb{C}}(t+i \tau, \zeta, y)=e^{i(t+i \tau) \Lambda}(\zeta, y): L^{2}(\partial \Omega) \rightarrow L^{2}\left((\partial \Omega)_{\mathbb{C}}\right)
$$

As discussed below, $\Lambda$ is an analytic pseudo-differential operator on $\partial \Omega$ when $(\Omega, \partial \Omega, g)$ is real analytic, and (5) is a Fourier integral operator with complex phase. (See [Bou2, Sj] for background on analytic pseudo-differential operators).

In the real analytic case, the Steklov eigenfunctions are real analytic on $\partial \Omega$ and have complex analytic extensions to $(\partial \Omega)_{\mathbb{C}}$. We then study their complex nodal sets

$$
\mathcal{N}_{\lambda}^{\mathbb{C}}=\left\{\zeta \in(\partial \Omega)_{\mathbb{C}}: \psi_{\lambda}^{\mathbb{C}}(\zeta)=0\right\}
$$

To prove Theorem 1, we use Crofton's formula and a multi-dimensional Jensen's formula to give an upper bound for $\mathcal{H}^{n-2}\left(\mathcal{N}_{\lambda}\right)$ in terms of the integral geometry of $\mathcal{N}_{\lambda}^{\mathbb{C}}$. The integral geometric approach to the upper bound 
is inspired by the classic paper of Donnelly-Fefferman [DG] (see also [Lin]). But, instead of doubling estimates or frequency function estimates, we use the Poisson wave kernel to obtain growth estimates on eigenfunctions, and then use results on pluri-subharmonic functions rather than functions of one complex variable to relate growth of zeros to growth of eigenfunctions. This approach was used in $[\mathrm{Z}]$ to prove equidistribution theorems for complex nodal sets when the geodesic flow is ergodic. The Poisson wave kernel approach works for Steklov eigenfunctions as well as Laplace eigenfunctions.

We first use the Poisson wave group (5) to analytically continue eigenfunctions in the form

$$
U_{\mathbb{C}}(i \tau) \psi_{j}(\zeta)=e^{-\tau \lambda_{j}} \psi_{j}^{\mathbb{C}}(\zeta) .
$$

We then use (7) to determine the growth properties of $\psi_{j}^{\mathbb{C}}(\zeta)$ in Grauert tubes of the complexification of $\partial \Omega$. The relevant notion of Grauert tube is the standard Grauert tube for $\partial \Omega$ with the metric $g_{\partial \Omega}$ induced by the ambient metric $g$ on $M$. This is because the principal symbol of $\Lambda$ is the same as the principal symbol of $\sqrt{\Delta_{\partial \Omega}}$.

Remark: A remark on notation: In [Z] we use $M$ to denote a Riemannian manifold, $M_{\epsilon}$ its Grauert tube of radius $\epsilon$ and $\partial M_{\epsilon}$ to denote the boundary of the Grauert tube of radius $\epsilon$. Since $\partial \Omega$ is the Riemannian manifold of interest here, we denote it by $M$,

$$
(M, g):=\left(\partial \Omega, g_{\partial \Omega}\right) .
$$

Thus the Grauert tube of radius $\tau$ of $(\partial \Omega)_{\mathbb{C}}$ is denoted $M_{\tau}$ and its boundary by $\partial M_{\tau}$, not to be confused with $\partial \Omega$. We also denote $m=\operatorname{dim} M=n-1$.

Because $U_{\mathbb{C}}(i \tau)$ is a Fourier integral operator with complex phase, it can only magnify the $L^{2}$ norm of $\psi_{j}$ by a power of $\lambda_{j}$. Hence the exponential $e^{\tau \lambda_{j}}$ dominates the $L^{2}$ norm on the boundary of the Grauert tube of radius $\tau$. We prove:

Proposition 2. Suppose $(\Omega, g, \partial \Omega)$ is real analytic. Let $\left\{\psi_{\lambda}\right\}$ be an eigenfunction of $\Lambda$ on $M=\partial \Omega$ of eigenvalue $\lambda$. Then

$$
\sup _{\zeta \in M_{\tau}}\left|\psi_{\lambda}^{\mathbb{C}}(\zeta)\right| \leq C \lambda^{\frac{m+1}{4}} e^{\tau \lambda}
$$

The proof follows from a standard cosine Tauberian result and the fact that the complexified Poisson kernel is a complex Fourier integral operator of finite order. This simple growth estimate replaces the doubling estimates of 
$[\mathrm{DF}]$ and $[\mathrm{BL}]$. It is closely related to growth estimates of $\Delta$-eigenfunctions in $[\mathrm{Z}, \mathrm{Z} 2, \mathrm{Z} 3]$.

For the precise statement that $U_{\mathbb{C}}(t+i \tau)$ is indeed a Fourier integral operator with complex phase, we refer to Theorem 3. It holds for positive elliptic analytic pseudo-differential operators whose principal symbol has convex sublevel sets. Since the principal symbol of $\Lambda$ is the same as for $\sqrt{\Delta_{\partial \Omega}}$, the proof is only a small modification of that in $[\mathrm{L}, \mathrm{Z} 2, \mathrm{St}]$ in the latter case and is omitted.

We thank Boris Hanin, G. Lebeau, Peng Zhou, Iosif Polterovich, Chris Sogge and particularly Y. Canzani for comments/corrections on earlier versions.

\section{Geometry and analysis of Grauert tubes}

We briefly review the geometry and analysis on Grauert tubes of real analytic Riemannian manifolds. We refer to [Z, Z2, GS1, GS2] for more detailed discussions.

\subsection{Analytic continuation to a Grauert tube}

A real analytic manifold $M$ always possesses a complexification $M_{\mathbb{C}}$, i.e. a complex manifold of which $M$ is a totally real submanifold. A real analytic Riemannian metric $g$ on $M$ determines a canonical plurisubharmonic function $\rho_{g}$ on $M_{\mathbb{C}}$; since the metric is fixed througout, we denote it simply by $\rho$. Its square-root $\sqrt{\rho}$ is known as the Grauert tube function; it equals $\sqrt{-r_{\mathbb{C}}^{2}(z, \bar{z})} / 2$ where $r_{\mathbb{C}}$ is the holomorphic extension of the distance function. The $(1,1)$ form $\omega=\omega_{\rho}:=i \partial \bar{\partial} \rho$ defines a Kähler metric on $M_{\mathbb{C}}$. The Grauert tubes $M_{\epsilon}:=\left\{z \in M_{\mathbb{C}}: \sqrt{\rho}(z)<\epsilon\right\}$ are strictly pseudo-convex domains in $M_{\mathbb{C}}$, whose boundaries are strictly pseudo-convex CR manifolds. We also denote the contact form of $\partial M_{\tau}$ by

$$
\alpha=\left.\frac{1}{i} \partial \rho\right|_{\partial M_{\tau}}=d^{c} \sqrt{\rho}
$$

The complexified exponential map

$$
(x, \xi) \in B_{\epsilon}^{*} M \rightarrow E(x, \xi):=\exp _{x}^{\mathbb{C}} \sqrt{-1} \xi \in M_{\epsilon}
$$

defines a symplectic diffeomorphism, where $B_{\epsilon}^{*} M \subset T^{*} M$ is the co-ball bundle of radius $\epsilon$, equipped with the standard symplectic structure, and where $M_{\epsilon}$ is equipped with $\omega_{\rho}$. The Grauert tube function $\sqrt{\rho}$ pulls back under 
$E$ to the metric norm function $|\xi|_{g}$. We emphase the setting $M_{\mathbb{C}}$ but it is equivalent to using $E$ to endow $B_{\epsilon}^{*} M$ with an adapted complex structure. We refer to [GS1, GS2, LS, GLS] for further discussion.

\subsection{Geodesic and Hamiltonian flows}

The microlocal analysis of the kernels (5) involves the complexification of the geodesic flow. We denote by $g^{t}$ the (real) homogeneous geodesic flow of $(M, g)$. It is the real analytic Hamiltonian flow on $T^{*} M \backslash 0_{M}$ generated by the Hamiltonian $|\xi|_{g}$ with respect to the standard symplectic form $\omega$. We also consider the Hamiltonian flow of $|\xi|_{g}^{2}$, which is real analytic on all of $T^{*} M$ and denote its Hamiltonian flow by $G^{t}$. In general, we denote by $\Xi_{H}$ the Hamiltonian vector field of a Hamiltonian $H$ and its flow by $\exp t \Xi_{H}$. Thus, we consider the Hamiltonian flows

$$
g^{t}=\exp t \Xi_{|\xi| g}, \quad \text { resp. } \quad G^{t}=\exp t \Xi_{|\xi|_{g}^{2}} .
$$

The exponential map is the map $\exp _{x}: T^{*} M \rightarrow M$ defined by $\exp _{x} \xi=$ $\pi G^{1}(x, \xi)$ where $\pi$ is the standard projection. Since $E^{*} \sqrt{\rho}=|\xi|, E^{*}$ conjugates the geodesic flow on $B^{*} M$ to the Hamiltonian flow $\exp t \Xi_{\sqrt{\rho}}$ of $\sqrt{\rho}$ with respect to $\omega$, i.e.

$$
E\left(g^{t}(x, \xi)\right)=\exp t \Xi_{\sqrt{\rho}}\left(\exp _{x} i \xi\right)
$$

\subsection{Szegö kernel and analytic continuation of the Poisson kernel}

We denote by $\mathcal{O}^{s+\frac{m-1}{4}}\left(\partial M_{\tau}\right)$ the Sobolev spaces of CR holomorphic functions on the boundaries of the strictly pseudo-convex domains $M_{\tau}$, i.e.

$$
\mathcal{O}^{s+\frac{m-1}{4}}\left(\partial M_{\tau}\right)=W^{s+\frac{m-1}{4}}\left(\partial M_{\tau}\right) \cap \mathcal{O}\left(\partial M_{\tau}\right),
$$

where $W^{s}$ is the $s$ th Sobolev space and where $\mathcal{O}\left(\partial M_{\tau}\right)$ is the space of boundary values of holomorphic functions. The inner product on $\mathcal{O}^{0}\left(\partial M_{\tau}\right)$ is with respect to the Liouville measure or contact volume form

$$
d \mu_{\tau}:=\alpha \wedge \omega^{m-1},
$$

on $\partial M_{\tau}$.

The study of norms of complexified eigenfunctions is related to the study of the Szegö kernels $\Pi_{\tau}$ of $M_{\tau}$, namely the orthogonal projections 


$$
\Pi_{\tau}: L^{2}\left(\partial M_{\tau}, d \mu_{\tau}\right) \rightarrow \mathcal{O}^{0}\left(\partial M_{\tau}, d \mu_{\tau}\right)
$$

onto the Hardy space of boundary values of holomorphic functions in $M_{\tau}$ which belong to $L^{2}\left(\partial M_{\tau}, d \mu_{\tau}\right)$. The Szegö projector $\Pi_{\tau}$ is a complex Fourier integral operator with a positive complex canonical relation. The real points of its canonical relation form the graph $\Delta_{\Sigma}$ of the identity map on the symplectic cone $\Sigma_{\tau} \subset T^{*} \partial M_{\tau}$ defined by the spray

$$
\Sigma_{\tau}=\left\{\left(\zeta, r d^{c} \sqrt{\rho}(\zeta)\right): r \in \mathbb{R}_{+}\right\} \subset T^{*}\left(\partial M_{\tau}\right)
$$

of the contact form $d^{c} \sqrt{\rho}$. There exists a symplectic equivalence (cf. [GS2])

$$
\iota_{\tau}: T^{*} M-0 \rightarrow \Sigma_{\tau}, \quad \iota_{\tau}(x, \xi)=\left(E\left(x, \tau \frac{\xi}{|\xi|}\right),|\xi| d^{c} \sqrt{\rho}_{E\left(x, \tau \frac{\xi}{|\xi|}\right.}\right) .
$$

\subsection{Analytic continuation of the Poisson wave kernel}

The wave group generated by $\Lambda$ on $M=\partial \Omega$ is the unitary group $U(t)=e^{i t \Lambda}$. Its kernel $U(t, x, y)$ solves the 'half-wave equation',

$$
\left(\frac{1}{i} \frac{\partial}{\partial t}-\Lambda_{x}\right) U(t, x, y)=0, \quad U(0, x, y)=\delta_{y}(x) .
$$

Here, $\Lambda_{x}$ means that $\Lambda$ is applied in the $x$ variable. In the real domain it is well known [Hor, DG] that $U(t, x, y)$ is the Schwartz kernel of a Fourier integral operator,

$$
U(t, x, y) \in I^{-1 / 4}(\mathbb{R} \times M \times M, \Gamma)
$$

with underlying canonical relation

$$
\Gamma=\left\{(t, \tau, x, \xi, y, \eta): \tau+|\xi|=0, g^{t}(x, \xi)=(y, \eta)\right\} \subset T^{*} \mathbb{R} \times T^{*} M \times T^{*} M .
$$

The Poisson-wave kernel is the analytic continuation $U(t+i \tau, x, y)$ of the wave kernel with respect to time, $t \rightarrow t+i \tau \in \mathbb{R} \times \mathbb{R}_{+}$. For $t=0$ and for $\tau>0$, we obtain the Poisson semi-group $U(i \tau)=e^{-\tau \Lambda}$. For general $t+i \tau$, 
the Poisson-wave kernel has the eigenfunction expansion,

$$
U(i \tau, x, y)=\sum_{j} e^{i(t+i \tau) \lambda_{j}} \psi_{\lambda_{j}}(x) \psi_{\lambda_{j}}(y) .
$$

As stated in Theorem 3 in the introduction, the Poisson-wave kernel $U(t+i \tau, x, y)$ admits an analytic continuation $U_{\mathbb{C}}(t+i \tau, \zeta, y)$ in the first variable to $M_{\tau} \times M$.

Theorem 3. Let $U(t)$ be the wave group of the Dirichlet to Neumann operator $\Lambda$ on $M=\partial \Omega$ as above. Then $\Pi_{\epsilon} \circ U(i \epsilon): L^{2}(M) \rightarrow \mathcal{O}\left(\partial M_{\epsilon}\right)$ is a Fourier integral operator with complex phase of order $-\frac{m-1}{4}$ associated to the canonical relation

$$
\Gamma=\left\{\left(y, \eta, \iota_{\epsilon}(y, \eta)\right\} \subset T^{*} \partial M_{\epsilon} \times \Sigma_{\epsilon} .\right.
$$

Moreover, for any s,

$$
\Pi_{\epsilon} \circ U(i \epsilon): W^{s}(M) \rightarrow \mathcal{O}^{s+\frac{m-1}{4}}\left(\partial M_{\epsilon}\right)
$$

is a continuous isomorphism.

This statement is asserted by Boutet de Monvel in [Bou, Bou2] for any real analytic positive elliptic pseudo-differential operator for which the sublevel sets $\left\{\xi \in T_{x}^{*} M: p(x, \xi) \leq 1\right\}$ are strictly convex, and has been accepted since then as an established fact (see for instance [GS1, GS2]). The proof was only sketched in [Bou, Bou2], and the first complete proofs appeared only recently in $[\mathrm{Z} 2, \mathrm{~L}, \mathrm{St}]$ for the special case of the wave group of a Riemannian manifold without boundary. Essentially the same proof applies to the wave group of $\Lambda$ as well because $\sqrt{\Delta_{\partial M}}$ and $\Lambda$ only differ by an analytic pseudo-differential operator of order zero, as follows from the fact that the principal symbol of $\Lambda$,

$$
\sigma_{\Lambda}: T^{*} \partial \Omega \rightarrow \mathbb{R}, \quad \sigma_{\Lambda}(x, \xi)=|\xi|_{g_{\partial}},
$$

is the same as for the Laplacian $\Delta_{\partial}$ of the boundary $\left(\partial \Omega, g_{\partial}\right)$. In fact, the complete symbol of $\Lambda$ is calculated in [LU] (see also [PS]).

\section{Growth of complexified eigenfunctions proof of Proposition 2}

We further need to generalize sup norm estimates of complexified eigenfunctions in $[\mathrm{Z} 2]$ to the $\Lambda$-eigenfunctions. 
As in [Z2, Z3] we prove Proposition 2 by introducing the 'tempered' spectral projections

$$
P_{I_{\lambda}}^{\tau}(\zeta, \bar{\zeta})=\sum_{j: \lambda_{j} \in I_{\lambda}} e^{-2 \tau \lambda_{j}}\left|\psi_{\lambda_{j}}^{\mathbb{C}}(\zeta)\right|^{2}, \quad(\sqrt{\rho}(\zeta) \leq \tau),
$$

where $I_{\lambda}$ could be a short interval $[\lambda, \lambda+1]$ of frequencies or a long window $[0, \lambda]$. Exactly as in [Z2] but with the wave group of $\Lambda$ replacing the wave group of $\sqrt{\Delta}$, we prove

$$
P_{[0, \lambda]}^{\tau}(\zeta, \bar{\zeta})=(2 \pi)^{-m}\left(\frac{\lambda}{\sqrt{\rho}}\right)^{\frac{m-1}{2}}\left(\frac{\lambda}{(m-1) / 2+1}+O(1)\right), \quad \zeta \in \partial M_{\tau}
$$

We then obtain

Corollary 4. Let $\psi_{\lambda}$ be an eigenfunction of $\Lambda$ as above. Then there exists $C>0$ so that for all $\sqrt{\rho}(\zeta)=\tau$,

$$
C \lambda^{-\frac{m-1}{2}} e^{\tau \lambda} \leq \sup _{\zeta \in M_{\tau}}\left|\psi_{\lambda}^{\mathbb{C}}(\zeta)\right| \leq C \lambda^{\frac{m-1}{4}+\frac{1}{2}} e^{\tau \lambda} .
$$

The upper bound is not sharp but is sufficient for our purposes. The lower bound is not used in the nodal analysis.

\subsection{Proof of the local Weyl law}

We only sketch the proof for the sake of completeness, since it is essentially the same as in [Z, Z2, Z3] and closely follows [DG]. The novelty is that we apply the argument of $[\mathrm{DG}]$ to the analytically continued parametrix.

By [Hor, DG] the positive elliptic first order pseudo-differential operator $\Lambda$ generates a wave group which has a parametrix of the form,

$$
U(t, x, y)=\int_{T_{y}^{*} M} e^{i t|\xi|_{g_{y}}} e^{i\left\langle\xi, \exp _{y}^{-1}(x)\right\rangle} A(t, x, y, \xi) d \xi
$$

similar to that of the wave kernel of $M=\partial \Omega$, since $\Lambda=\sqrt{\Delta_{M}}+Q$ where $Q$ is an analytic pseudo-differential operator of order zero. Here, $|\xi|_{g_{x}}$ is the metric norm function at $x$, and where $A(t, x, y, \xi)$ is a polyhomogeneous amplitude of order 0 which is supported near the diagonal. The amplitude is different from that of the wave kernel since the transport equations involve $Q$. 
By Theorem 3, the wave group and parametrix may be analytically continued. To obtain uniform asymptotics, we use the analytic continuation of the Hörmander parametrix (23). We choose local coordinates near $x$ and write $\exp _{x}^{-1}(y)=\Phi(x, y)$ in these local coordinates for $y$ near $x$, and write the integral $T_{y}^{*} M$ as an integral over $\mathbb{R}^{m}$ in these coordinates. The holomorphic extension of the parametrix to the Grauert tube $|\zeta|<\tau$ at time $t+2 i \tau$ has the form

$$
U_{\mathbb{C}}(t+2 i \tau, \zeta, \bar{\zeta})=\int_{\mathbb{R}^{m}} e^{(i t-2 \tau)|\xi|_{g_{y}}} e^{i\langle\xi, \Phi(\zeta, \bar{\zeta})\rangle} A(t, \zeta, \bar{\zeta}, \xi) d \xi,
$$

where $A$ is the analytic extensions of the real analytic $A$ and $\Phi(\zeta, \bar{\zeta})$ is the analytic extension of $\exp _{y}^{-1}(x)$.

We introduce a cutoff function $\chi \in \mathcal{S}(\mathbb{R})$ with $\hat{\chi} \in C_{0}^{\infty}$ supported in sufficiently small neighborhood of 0 so that no other singularities of $U_{\mathbb{C}}(t+$ $2 i \tau, \zeta, \bar{\zeta})$ lie in its support. We also assume $\hat{\chi} \equiv 1$ in a smaller neighborhood of 0 . We then change variables $\xi \rightarrow \lambda \xi$ and apply the complex stationary phase to the integral,

$$
\begin{aligned}
& \int_{\mathbb{R}} \hat{\chi}(t) e^{-i \lambda t} U_{\mathbb{C}}(t+2 i \tau, \zeta, \bar{\zeta}) d t \\
= & \lambda^{m} \int_{0}^{\infty} \int_{\mathbb{R}} \hat{\chi}(t) e^{-i \lambda t} \\
& \int_{S^{m-1}} e^{(i t-2 \tau) \lambda r} e^{i r \lambda\langle\omega, \Phi(\zeta, \bar{\zeta})\rangle} A(t, \zeta, \bar{\zeta}, \lambda r \omega) r^{m-1} d r d t d \omega .
\end{aligned}
$$

The resulting integral (25) is a semi-classical Fourier integral distribution with a complex phase, the same phase as in the pure Riemannian case treated in [Z2]. Hence the stationary phase calculation is essentially the same as in section 9.1 of [Z2]. We first integrate over $d \omega$ and find that there are two stationary phase points, one giving an exponentially decaying amplitude of order $e^{-2 \lambda \tau r}$ and one for which the critical value is $2 \lambda \tau r$. It cancels the term $-2 \tau \lambda r$ coming from the factor $e^{(i t-2 \tau) \lambda r}$. We then apply stationary phase to the resulting integral over $(t, r)$ with phase $t(r-1)$. The critical set consists of $r=1, t=0$. The phase is clearly non-degenerate with Hessian determinant one and inverse Hessian operator $D_{\theta, t}^{2}$. Taking into account the factor of $\lambda^{-1}$ from the change of variables, the stationary phase expansion gives

$$
\sum_{j} \psi\left(\lambda-\lambda_{j}\right) e^{-2 \tau \lambda_{j}}\left|\psi_{j}^{\mathbb{C}}(\zeta)\right|^{2} \sim \sum_{k=0}^{\infty} \lambda^{\frac{m-1}{2}-k} \omega_{k}(\tau ; \zeta),
$$


where the coefficients $\omega_{k}(\tau, \zeta)$ are smooth for $\zeta \in \partial M_{\tau}$. The Weyl asymptotics then follows from the standard cosine Tauberian theorem, as in [DG] or [Z2] (loc. cit.).

\section{Proof of Theorem 1}

We start with the integral geometric approach of [DF] (Lemma 6.3) (see also [Lin] (3.21)). There exists a "Crofton formula" in the real domain which bounds the local nodal hypersurface volume above,

$$
\mathcal{H}^{m-1}\left(\mathcal{N}_{\varphi_{\lambda}} \cap U\right) \leq C_{L} \int_{\mathcal{L}} \#\left\{\mathcal{N}_{\varphi_{\lambda}} \cap \ell\right\} d \mu(\ell) .
$$

Thus, $\mathcal{H}^{m-1}\left(\mathcal{N}_{\varphi_{\lambda}} \cap U\right)$ is bounded above by a constant $C_{L}$ times the average over all line segments of length $L$ in a local coordinate patch $U$ of the number of intersection points of the line with the nodal hypersurface. The measure $d \mu_{L}$ is known as the 'kinematic measure' in the Euclidean setting [F] (Chapter 3); see also Theorem 5.5 of $[\mathrm{AP}]$. We will be using geodesic segments of fixed length $L$ rather than line segments, and parametrize them by $S^{*} M \times[0, L]$, i.e. by their intial data and time. Then $d \mu_{\ell}$ is essentially Liouville measure $d \mu_{L}$ on $S^{*} M$ times $d t$.

The complexification of a real line $\ell=x+\mathbb{R} v$ with $x, v \in \mathbb{R}^{m}$ is $\ell_{\mathbb{C}}=$ $x+\mathbb{C} v$. Since the number of intersection points (or zeros) only increases if we count complex intersections, we have

$$
\int_{\mathcal{L}} \#\left(\mathcal{N}_{\varphi_{\lambda}} \cap \ell\right) d \mu(\ell) \leq \int_{\mathcal{L}} \#\left(\mathcal{N}_{\varphi_{\lambda}}^{\mathbb{C}} \cap \ell_{\mathbb{C}}\right) d \mu(\ell) .
$$

Note that this complexification is quite different from using intersections with all complex lines to measure complex nodal volumes. If we did that, we would obtain a similar upper bound on the complex hypersurface volume of the complex nodal set. But it would not give an upper bound on the real nodal volume and indeed would the complex volume tends to zero as one shrinks the Grauert tube radius to zero, while (28) stays bounded below.

Hence to prove Theorem 1 it suffices to show

Lemma 5. We have,

$$
\left.\mathcal{H}^{m-1}\left(\mathcal{N}_{\varphi_{\lambda}}\right) \leq C_{L} \int_{\mathcal{L}} \#\left(\mathcal{N}_{\varphi_{\lambda}}\right)^{\mathbb{C}} \cap \ell_{\mathbb{C}}\right) d \mu(\ell) \leq C \lambda .
$$

We now sketch the proofs of these results using a somewhat novel approach to the integral geometry and complex analysis. 


\subsection{Background on hypersurfaces and geodesics}

The proof of the Crofton formula given below in Proposition 9 involves the geometry of geodesics and hypersurfaces. To prepare for it we provide the relevant background.

As above, we denote by $d \mu_{L}$ the Liouville measure on $S^{*} M$. We also denote by $\omega$ the standard symplectic form on $T^{*} M$ and by $\alpha$ the canonical one form. Then $d \mu_{L}=\omega^{m-1} \wedge \alpha$ on $S^{*} M$. Indeed, $d \mu_{L}$ is characterized by the formula $d \mu_{L} \wedge d H=\omega^{m}$, where $H(x, \xi)=|\xi|_{g}$. We take the interior product $\iota_{\Xi_{H}}$ with the Hamilton vector field $\Xi_{H}$ on both sides, and the identity follows from the fact that $\alpha\left(\Xi_{H}\right)=\sum_{j} \xi_{j} \frac{\partial H}{\partial \xi_{j}}=H=1$ on $S^{*} M$, since $H$ is homogeneous of degree one. Henceforth we denote by $\Xi=\Xi_{H}$ the generator of the geodesic flow.

Let $N \subset M$ be a smooth hypersurface in a Riemannian manifold $(M, g)$. We denote by $T_{N}^{*} M$ the of covectors with footpoint on $N$ and $S_{N}^{*} M$ the unit covectors along $N$. We introduce Fermi normal coordinates $\left(s, y_{m}\right)$ along $N$, where $s$ are coordinates on $N$ and $y_{m}$ is the normal coordinate, so that $y_{m}=0$ is a local defining function for $N$. We also let $\sigma, \xi_{m}$ be the dual symplectic Darboux coordinates. Thus the canonical symplectic form is $\omega_{T^{*} M}=d s \wedge d \sigma+d y_{m} \wedge d \xi_{m}$. Let $\pi: T^{*} M \rightarrow M$ be the natural projection. For notational simplicity we denote $\pi^{*} y_{m}$ by $y_{m}$ as functions on $T^{*} M$. Then $y_{m}$ is a defining function of $T_{N}^{*} M$.

The hypersurface $S_{N}^{*} M \subset S^{*} M$ is a kind of Poincaré section or symplectic transversal to the orbits of $G^{t}$, i.e. is a symplectic transversal away from the (at most codimension one) set of $(y, \eta) \in S_{N}^{*} M$ for which $\Xi_{y, \eta} \in$ $T_{y, \eta} S_{N}^{*} M$, where as above $\Xi$ is the generator of the geodesic flow. More precisely,

Lemma 6. The restriction $\left.\omega\right|_{S_{N}^{*} M}$ is symplectic on $S_{N}^{*} M \backslash S^{*} N$.

Indeed, $\left.\omega\right|_{S_{N}^{*} M}$ is symplectic on $T_{y, \eta} S^{*} N$ as long as $T_{y, \eta} S_{N}^{*} M$ is transverse to $\Xi_{y, \eta}$, since $\operatorname{ker}\left(\left.\omega\right|_{S^{*} M}\right)=\mathbb{R} \Xi$. But $S^{*} N$ is the set of points of $S_{N}^{*} M$ where $\Xi \in T S_{N}^{*} M$, i.e. where $S_{N}^{*} M$ fails to be transverse to $G^{t}$. Indeed, transversality fails when $\Xi\left(y_{m}\right)=d y_{m}(\Xi)=0$, and ker $d y_{m} \cap \operatorname{ker} d H=$ $T S_{N}^{*} M$. One may also see it in Riemannian terms as follows: the generator $\Xi_{y, \eta}$ is the horizontal lift $\eta^{h}$ of $\eta$ to $(y, \eta)$ with respect to the Riemannian connection on $S^{*} M$, where we freely identify covectors and vectors by the metric. Lack of transversality occurs when $\eta^{h}$ is tangent to $T_{(y, \eta)}\left(S_{N}^{*} M\right)$. The latter is the kernel of $d y_{m}$. But $d y_{m}\left(\eta^{h}\right)=d y_{m}(\eta)=0$ if and only if $\eta \in T N$. 
It follows from Lemma 6 that the symplectic volume form of $S_{N}^{*} M \backslash S^{*} N$ is $\left.\omega^{m-1}\right|_{S_{N}^{*} M}$. The following Lemma gives a useful alternative formula:

Lemma 7. Define

$$
d \mu_{L, N}=\left.\iota \Xi d \mu_{L}\right|_{S_{N}^{*} M}
$$

where as above, $d \mu_{L}$ is Liouville measure on $S^{*} M$. Then

$$
d \mu_{L, N}=\left.\omega^{m-1}\right|_{S_{N}^{*} M}
$$

Indeed, $d \mu_{L}=\omega^{m-1} \wedge \alpha$, and $\iota_{\Xi} d \mu_{L}=\omega^{m-1}$.

Corollary 8. $\mathcal{H}^{m-1}(N)=\frac{1}{\beta_{m}} \int_{S_{N}^{*} M}\left|\omega^{m-1}\right|$.

\subsection{Hausdorff measure and Crofton formula for real geodesic arcs}

First we sketch a proof of the integral geometry estimate using geodesic arcs rather than local coordinate line segments. For background on integral geometry and Crofton type formulae we refer to $[\mathrm{AB}, \mathrm{AP}]$. As explained there, a Crofton formula arises from a double fibration

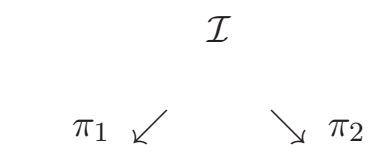

$\Gamma$

$B$,

where $\Gamma$ parametrizes a family of submanifolds $B_{\gamma}$ of $B$. The points $b \in B$ then parametrize a family of submanifolds $\Gamma_{b}=\left\{\gamma \in \Gamma: b \in B_{\gamma}\right\}$ and the top space is the incidence relation in $B \times \Gamma$ that $b \in B_{\gamma}$.

We would like to define $\Gamma$ as the space of geodesics of $(M, g)$, i.e. the space of orbits of the geodesic flow on $S^{*} M$. Heuristically, the space of geodesics is the quotient space $S^{*} M / \mathbb{R}$ where $\mathbb{R}$ acts by the geodesic flow $G^{t}$ (i.e. the Hamiltonian flow of $H$ ). Of course, for a general (i.e. non-Zoll) $(M, g)$ the 'space of geodesics' is not a Hausdorff space and so we do not have a simple analogue of the space of lines in $\mathbb{R}^{n}$. Instead we consider the space $\mathcal{G}_{T}$ of geodesic arcs of length $T$. If we only use partial orbits of length $T$, no two partial orbits are equivalent and the space of geodesic arcs $\gamma_{x, \xi}^{T}$ of length $T$ is simply parametrized by $S^{*} M$. Hence we let $B=S^{*} M$ and also $\mathcal{G}_{T} \simeq S^{*} M$. The fact that different arcs of length $T$ of the same geodesic are distinguished leads to some redundancy. 
In the following, let $L_{1}$ denote the length of the shortest closed geodesic of $(M, g)$.

Proposition 9. Let $N \subset M$ be any smooth hypersurface ${ }^{1}$, and let $S_{N}^{*} M$ denote the unit covers to $M$ with footpoint on $N$. Then for $0<T<L_{1}$,

$$
\mathcal{H}^{m-1}(N)=\frac{1}{\beta_{m} T} \int_{S^{*} M} \#\left\{t \in[-T, T]: G^{t}(x, \omega) \in S_{N}^{*} M\right\} d \mu_{L}(x, \omega),
$$

where $\beta_{m}$ is $2(m-1)$ ! times the volume of the unit ball in $\mathbb{R}^{m-2}$.

Proof. By Corollary 8, the Hausdorff measure of $N$ is given by

$$
\mathcal{H}^{m-1}(N)=\frac{1}{\beta_{m}} \int_{S_{N}^{*} M}\left|\omega^{m-1}\right| .
$$

We use the Lagrange (or more accurately, Legendre) immersion,

$$
\iota: S^{*} M \times \mathbb{R} \rightarrow S^{*} M \times S^{*} M, \iota(x, \omega, t)=\left(x, \omega, G^{t}(x, \omega)\right),
$$

where as above, $G^{t}$ is the geodesic flow (11). We also let $\pi: T^{*} M \rightarrow M$ be the standard projection. We restrict $\iota$ to $S^{*} M \times[-T, T]$ and define the incidence relation

$$
\mathcal{I}_{T}=\left\{((y, \eta),(x, \omega), t) \subset S^{*} M \times S^{*} M \times[-T, T]:(y, \eta)=G^{t}(x, \omega)\right\},
$$

which is isomorphic to $[-T, T] \times S^{*} M$ under $\iota$. We form the diagram

$$
\begin{aligned}
& \mathcal{I}_{T} \simeq S^{*} M \times[-T, T] \\
& \pi_{1} \swarrow \quad \searrow \pi_{2} \\
& S^{*} M \simeq \mathcal{G}_{T} \\
& S^{*} M
\end{aligned}
$$

using the two natural projections, which in the local parametrization take the form

$$
\pi_{1}(t, x, \xi)=G^{t}(x, \xi), \quad \pi_{2}(t, x, \xi)=(x, \xi) .
$$

As noted above, the bottom left $S^{*} M$ should be thought of as the space of geodesic arcs. The fiber

$$
\pi_{1}^{-1}(y, \eta)=\left\{(t, x, \xi) \in[-T, T] \times S^{*} M: G^{t}(x, \xi)=(y, \eta)\right\} \simeq \gamma_{(y, \eta)}^{T}
$$

\footnotetext{
${ }^{1}$ The same formula is true if $N$ has a singular set $\Sigma$ with $\mathcal{H}^{m-2}(\Sigma)<\infty$
} 
may be identified with the geodesic segment through $(y, \eta)$ and the fiber $\pi_{2}^{-1}(x, \omega) \simeq[-T, T]$.

We 'restrict' the diagram above to $S_{N}^{*} M$ :

$$
\mathcal{I}_{T} \simeq S_{N}^{*} M \times[-T, T]
$$

$$
\begin{array}{rll}
\pi_{1} \swarrow & \searrow \pi_{2} & \\
\left(S_{N}^{*} M\right)_{T} & & S_{N}^{*} M,
\end{array}
$$

where

$$
\left(S_{N}^{*} M\right)_{T}=\pi_{1} \pi_{2}^{-1}\left(S_{N}^{*} M\right)=\bigcup_{|t|<T} G^{t}\left(S_{N}^{*} M\right) .
$$

We define the Crofton density $\varphi_{T}$ on $S_{N}^{*} M$ corresponding to the diagram (30) [AP] (section 4) by

$$
\varphi_{T}=\left(\pi_{2}\right)_{*} \pi_{1}^{*} d \mu_{L}
$$

Since the fibers of $\pi_{2}$ are 1-dimensional, $\varphi_{T}$ is a differential form of dimension $2 \operatorname{dim} M-2$ on $S^{*} M$. To make it smoother, we can introduce a smooth cutoff $\chi$ to $(-1,1)$, equal to 1 on $\left(-\frac{1}{2}, \frac{1}{2}\right)$, and use $\chi_{T}(t)=\chi\left(\frac{t}{T}\right)$. Then $\pi_{1}^{*}\left(d \mu_{L} \otimes \chi_{T} d t\right)$ is a smooth density on $\mathcal{I}_{T}$.

Lemma 10. The Crofton density (31) is given by, $\varphi_{T}=T d \mu_{L, N}$

Proof. In (30) we defined the map $\pi_{1}:(y, \eta, t) \in S_{N}^{*} M \times[-T, T] \rightarrow G^{t}(y, \eta) \in$ $\left(S^{*} M\right)_{\epsilon}$. We first claim that $\pi_{1}^{*} d \mu_{L}=d \mu_{L, N} \otimes d t$. This is essentially the same as Lemma 7 . Indeed, $d \pi_{1}\left(\frac{\partial}{\partial t}\right)=\Xi$, hence $\left.\iota_{\frac{\partial}{\partial t}} \pi_{1}^{*} d \mu_{L}\right|_{(t, y, \eta)}=\left(G^{t}\right)^{*} \omega^{m-1}=$ $\left.\omega^{m-1}\right|_{T_{y, \eta} S_{N}^{*} M}$.

Combining Lemma 10 with (29) gives

$$
\int_{S_{N}^{*} M} \varphi_{T}=\int_{\pi_{2}^{-1}\left(S_{N}^{*} M\right)} d \mu_{L}=T \beta_{m} \mathcal{H}^{m-1}(N) .
$$

We then relate the integral on the left side to numbers of intersections of geodesic arcs with $N$. The relation is given by the co-area formula: if $f: X \rightarrow Y$ is a smooth map of manifolds of the same dimension and if $\Phi$ is a smooth density on $Y$, and if $\#\left\{f^{-1}(y)\right\}<\infty$ for every regular value $y$, 
then

$$
\int_{X} f^{*} \Phi=\int_{Y} \#\left\{f^{-1}(y)\right\} \Phi
$$

If we set set $X=\pi_{2}^{-1}\left(S_{N}^{*} M\right), Y=S^{*} M$, and $f=\left.\pi_{1}\right|_{\pi_{2}^{-1}\left(S_{N}^{*} M\right)}$ then the coarea formula gives,

$$
\int_{\pi_{2}^{-1}\left(S_{N}^{*} M\right)} \pi_{1}^{*} d \mu_{L}=\int_{S^{*} M} \#\left\{t \in[-T, T]: G^{t}(x, \omega) \in S_{N}^{*} M\right\} d \mu_{L}(x, \omega) .
$$

Combining (32) and (33) gives the result stated in Proposition 9,

$$
T \beta_{m} \mathcal{H}^{m-1}(N)=\int_{S^{*} M} \#\left\{t \in[-T, T]: G^{t}(x, \omega) \in S_{N}^{*} M\right\} d \mu_{L}(x, \omega)
$$

\subsection{Proof of Lemma 5}

The next step is to complexify.

Proof. We complexify the Lagrange immersion $\iota$ from a line (segment) to a strip in $\mathbb{C}$ : Define

$$
F: S_{\epsilon} \times S^{*} M \rightarrow M_{\mathbb{C}}, \quad F(t+i \tau, x, v)=\exp _{x}(t+i \tau) v, \quad(|\tau| \leq \epsilon)
$$

By definition of the Grauert tube, $\psi$ is surjective onto $M_{\epsilon}$. For each $(x, v) \in$ $S^{*} M$

$$
F_{x, v}(t+i \tau)=\exp _{x}(t+i \tau) v
$$

is a holomorphic strip. Here, $S_{\epsilon}=\{t+i \tau \in \mathbb{C}:|\tau| \leq \epsilon\}$. We also denote by $S_{\epsilon, L}=\{t+i \tau \in \mathbb{C}:|\tau| \leq \epsilon,|t| \leq L\}$.

Since $F_{x, v}$ is a holomorphic strip,

$$
\begin{aligned}
F_{x, v}^{*}\left(\frac{1}{\lambda} d d^{c} \log \left|\psi_{j}^{\mathbb{C}}\right|^{2}\right) & =\frac{1}{\lambda} d d_{t+i \tau}^{c} \log \left|\psi_{j}^{\mathbb{C}}\right|^{2}\left(\exp _{x}(t+i \tau) v\right) \\
& =\frac{1}{\lambda} \sum_{t+i \tau: \psi_{j}^{\mathbb{C}}\left(\exp _{x}(t+i \tau) v\right)=0} \delta_{t+i \tau} .
\end{aligned}
$$


Put:

$$
\begin{aligned}
& \mathcal{A}_{L, \epsilon}\left(\frac{1}{\lambda} d d^{c} \log \left|\psi_{j}^{\mathbb{C}}\right|^{2}\right) \\
= & \frac{1}{\lambda} \int_{S^{*} M} \int_{S_{\epsilon, L}} d d_{t+i \tau}^{c} \log \left|\psi_{j}^{\mathbb{C}}\right|^{2}\left(\exp _{x}(t+i \tau) v\right) d \mu_{L}(x, v) .
\end{aligned}
$$

A key observation of $[\mathrm{DF}, \mathrm{Lin}]$ is that

$$
\#\left\{\mathcal{N}_{\lambda}^{\mathbb{C}} \cap F_{x, v}\left(S_{\epsilon, L}\right)\right\} \geq \#\left\{\mathcal{N}_{\lambda}^{\mathbb{R}} \cap F_{x, v}\left(S_{0, L}\right)\right\},
$$

since every real zero is a complex zero. It follows then from Proposition 9 (with $N=\mathcal{N}_{\lambda}$ ) that

$$
\begin{aligned}
\mathcal{A}_{L, \epsilon}\left(\frac{1}{\lambda} d d^{c} \log \left|\psi_{j}^{\mathbb{C}}\right|^{2}\right) & =\frac{1}{\lambda} \int_{S^{*} M} \#\left\{\mathcal{N}_{\lambda}^{\mathbb{C}} \cap F_{x, v}\left(S_{\epsilon, L}\right)\right\} d \mu(x, v) \\
& \geq \frac{1}{\lambda} \mathcal{H}^{m-1}\left(\mathcal{N}_{\psi_{\lambda}}\right)
\end{aligned}
$$

Hence to obtain an upper bound on $\frac{1}{\lambda} \mathcal{H}^{m-1}\left(\mathcal{N}_{\psi_{\lambda}}\right)$ it suffices to prove that there exists $M<\infty$ so that

$$
\mathcal{A}_{L, \epsilon}\left(\frac{1}{\lambda} d d^{c} \log \left|\psi_{j}^{\mathbb{C}}\right|^{2}\right) \leq M
$$

To prove (37), we observe that since $d d_{t+i \tau}^{c} \log \left|\psi_{j}^{\mathbb{C}}\right|^{2}\left(\exp _{x}(t+i \tau) v\right)$ is a positive $(1,1)$ form on the strip, the integral over $S_{\epsilon}$ is only increased if we integrate against a positive smooth test function $\chi_{\epsilon} \in C_{c}^{\infty}(\mathbb{C})$ which equals one on $S_{\epsilon, L}$ and vanishes off $S_{2 \epsilon, L}$. Integrating by parts the $d d^{c}$ onto $\chi_{\epsilon}$, we have

$$
\begin{aligned}
& \mathcal{A}_{L, \epsilon}\left(\frac{1}{\lambda} d d^{c} \log \left|\psi_{j}^{\mathbb{C}}\right|^{2}\right) \\
\leq & \frac{1}{\lambda} \int_{S^{*} M} \int_{\mathbb{C}} d d_{t+i \tau}^{c} \log \left|\psi_{j}^{\mathbb{C}}\right|^{2}\left(\exp _{x}(t+i \tau) v\right) \chi_{\epsilon}(t+i \tau) d \mu_{L}(x, v) \\
= & \frac{1}{\lambda} \int_{S^{*} M} \int_{\mathbb{C}} \log \left|\psi_{j}^{\mathbb{C}}\right|^{2}\left(\exp _{x}(t+i \tau) v\right) d d_{t+i \tau}^{c} \chi_{\epsilon}(t+i \tau) d \mu_{L}(x, v) .
\end{aligned}
$$

Now write $\log |x|=\log _{+}|x|-\log _{-}|x|$. Here $\log _{+}|x|=\max \{0, \log |x|\}$ and $\log _{-}|x|=\max \{0,-\log |x|\}$. Then we need upper bounds for

$$
\frac{1}{\lambda} \int_{S^{*} M} \int_{\mathbb{C}} \log _{ \pm}\left|\psi_{j}^{\mathbb{C}}\right|^{2}\left(\exp _{x}(t+i \tau) v\right) d d_{t+i \tau}^{c} \chi_{\epsilon}(t+i \tau) d \mu_{L}(x, v)
$$


For $\log _{+}$the upper bound is an immediate consequence of Proposition 2. For $\log _{-}$the bound is subtler: we need to show that $\left|\varphi_{\lambda}(z)\right|$ cannot be too small on too large a set. As we know from Gaussian beams, it is possible that $\left|\varphi_{\lambda}(x)\right| \leq C e^{-\delta \lambda}$ on sets of almost full measure in the real domain; we need to show that nothing worse can happen.

The map (10) is a diffeomorphism and since $B_{\epsilon}^{*} M=\bigcup_{0 \leq \tau \leq \epsilon} S_{\tau}^{*} M$ we also have that

$$
E: S_{\epsilon, L} \times S^{*} M \rightarrow M_{\tau}, \quad E(t+i \tau, x, v)=\exp _{x}(t+i \tau) v
$$

is a diffeomorphism for each fixed $t$. Hence by letting $t$ vary, $E$ is a smooth fibration with fibers given by geodesic arcs. Over a point $\zeta \in M_{\tau}$ the fiber of the map is a geodesic arc

$$
\left\{(t+i \tau, x, v): \exp _{x}(t+i \tau) v=\zeta, \quad \tau=\sqrt{\rho}(\zeta)\right\} .
$$

Pushing forward the measure $d d_{t+i \tau}^{c} \chi_{\epsilon}(t+i \tau) d \mu_{L}(x, v)$ under $E$ gives a measure $d \mu$ on $M_{\tau}$. We claim that

$$
\mu:=E_{*} d d_{t+i \tau}^{c} \chi_{\epsilon}(t+i \tau) d \mu_{L}(x, v)=\left(\int_{\gamma_{x, v}} \Delta_{t+i \tau} \chi_{\epsilon} d s\right) d V_{\omega},
$$

where $d V_{\omega}$ is the Kähler volume form $\frac{\omega^{m}}{m !}$ (see $\S 1.1$.)

In fact, $d \mu_{L}$ is equivalent under $E$ to the contact volume form $\alpha \wedge \omega_{\rho}^{m-1}$ where $\alpha=d^{c} \sqrt{\rho}$. Hence the claim amounts to saying that the Kähler volume form is $d \tau$ times the contact volume form. In particular it is a smooth (and of course signed) multiple $J$ of the Kähler volume form $d V_{\omega}$, and we do not need to know the coefficient function $J$ beyond that it is bounded above and below by constants independent of $\lambda$. We then have

$$
\begin{aligned}
& \int_{S^{*} M} \int_{\mathbb{C}} \log \left|\psi_{j}^{\mathbb{C}}\right|^{2}\left(\exp _{x}(t+i \tau) v\right) d d_{t+i \tau}^{c} \chi_{\epsilon}(t+i \tau) d \mu_{L}(x, v) \\
= & \int_{M_{\tau}} \log \left|\psi_{j}^{\mathbb{C}}\right|^{2} J d V .
\end{aligned}
$$

To complete the proof of (37) it suffices to prove that the right side is $\geq-C \lambda$ for some $C>0$.

We use the well-known

Lemma 11. (Hartog's Lemma; (see [HoI-IV, Theorem 4.1.9]): Let $\left\{u_{j}\right\}$ be a sequence of subharmonic functions in an open set $X \subset \mathbb{R}^{m}$ which have a 
uniform upper bound on any compact set. Then either $u_{j} \rightarrow-\infty$ uniformly on every compact set, or else there exists a subsequence $u_{j_{k}}$ which is convergent to some $u \in L_{l o c}^{1}(X)$. Further, $\limsup _{n} u_{n}(x) \leq u(x)$ with equality almost everywhere. For every compact subset $K \subset X$ and every continuous function $f$,

$$
\limsup _{n \rightarrow \infty} \sup _{K}\left(u_{n}-f\right) \leq \sup _{K}(u-f) .
$$

In particular, if $f \geq u$ and $\epsilon>0$, then $u_{n} \leq f+\epsilon$ on $K$ for $n$ large enough.

This Lemma implies the desired lower bound on (39): there exists $C>0$ so that

$$
\frac{1}{\lambda} \int_{M_{\tau}} \log \left|\psi_{\lambda}^{\mathbb{C}}\right| J d V \geq-C
$$

For if not, there exists a subsequence of eigenvalues $\lambda_{j_{k}}$ so that

$$
\frac{1}{\lambda_{j_{k}}} \int_{M_{\tau}} \log \left|\psi_{\lambda_{j_{k}}}^{\mathbb{C}}\right| J d V \rightarrow-\infty .
$$

By Proposition 2, $\left\{\frac{1}{\lambda_{j_{k}}} \log \left|\psi_{\lambda_{j_{k}}}^{\mathbb{C}}\right|\right\}$ has a uniform upper bound. Moreover the sequence does not tend uniformly to $-\infty$ since $\left\|\psi_{\lambda}^{\mathbb{C}}\right\|_{L^{2}(M)}=1$. It follows that a further subsequence tends in $L^{1}$ to a limit $u$ and by the dominated convergence theorem the limit of (40) along the sequence equals $\int_{M_{\tau}} u J d V \neq$ $-\infty$. This contradiction concludes the proof of (40), hence (37), and thus the theorem.

\section{References}

[AB] J. C. Alvarez Paiva and G. Berck, What is wrong with the Hausdorff measure in Finsler spaces. Adv. Math., 204 (2006), no. 2, 647-663.

[AP] J. C. Alvarez Paiva and E. Fernandes, Gelfand transforms and Crofton formulas. Selecta Math. (N.S.), 13 (2007), no. 3, 369-390.

[BL] K. Bellova and F. H. Lin, Nodal Sets of Steklov Eigenfunctions. Calc. Var. Partial Differential Equations, 54 (2015), no. 2, 22392268. arXiv: 1402.4323.

[B] R. L. Bishop, Infinitesimal convexity implies local convexity. Indiana Univ. Math. J., 24 (1974/75), 169-172. 
[Bou] L. Boutet de Monvel, Convergence dans le domaine complexe des séries de fonctions propres. C. R. Acad. Sci. Paris Sér. A-B, 287 (1978), no. 13, A855-A856.

[Bou2] L. Boutet de Monvel, Convergence dans le domaine complexe des séries de fonctions propres. Journées: Équations aux Dérivées Partielles (Saint-Cast, 1979), Exp. No. 3, 2 pp., École Polytech., Palaiseau, 1979.

[BK] L. Boutet de Monvel and P. Krée, Pseudo-differential operators and Gevrey classes. Ann. Inst. Fourier, 17 (1967), 295-323.

[DF] H. Donnelly and C. Fefferman, Nodal sets of eigenfunctions on Riemannian manifolds. Invent. Math., 93 (1988), no. 1, 161-183.

[DG] J. J. Duistermaat and V. Guillemin, The spectrum of positive elliptic operators and periodic bicharacteristics. Inv. Math., 24 (1975), 39-80.

[F] H. Federer, Geometric measure theory. Die Grundlehren der mathematischen Wissenschaften, Band 153 Springer-Verlag New York Inc., New York 1969.

[GPPS] A. Girouard, L. Parnovski, I. Polterovich and D. A. Sher, The Steklov spectrum of surfaces: asymptotics and invariants. Math. Proc. Camb. Philos. Soc., 157 (2014), 379-389. arXiv:1311. 5533.

[GLS] F. Golse, E. Leichtnam and M. Stenzel, Intrinsic microlocal analysis and inversion formulae for the heat equation on compact realanalytic Riemannian manifolds. Ann. Sci. École Norm. Sup. (4), 29 (1996), no. 6, 669-736.

[GS1] V. Guillemin and M. Stenzel, Grauert tubes and the homogeneous Monge-Ampère equation. J. Differential Geom., 34 (1991), no. 2, $561-570$.

[GS2] V. Guillemin and M. Stenzel, Grauert tubes and the homogeneous Monge-Ampère equation. II. J. Differential Geom., 35 (1992), no. 3, 627-641.

[Hor] L. Hörmander, The spectral function of an elliptic operator. Acta Math., 121 (1968), 193-218.

[HoI-IV] L. Hörmander, Theory of Linear Partial Differential Operators I$I V$. Springer-Verlag, New York (1985). 
[L] G. Lebeau, The complex Poisson kernel on a compact analytic Riemannian manifold. To appear in: Analytic and Algebraic Microlocal Analysis, Springer Verlag.

[LS] L. Lempert and R. Szöke, Global solutions of the homogeneous complex Monge-Ampère equation and complex structures on the tangent bundle of Riemannian manifolds. Math. Ann., 290 (1991), no. 4, 689-712.

[LU] J. M. Lee and G. Uhlmann, Determining anisotropic real-analytic conductivities by boundary measurements. Comm. Pure Appl. Math., 42 (1989), no. 8, 1097-1112.

[Lin] F. H. Lin, Nodal sets of solutions of elliptic and parabolic equations. Comm. Pure Appl. Math., 44 (1991), no. 3, 287-308.

[PS] I. Polterovich and D. A. Sher, Heat invariants of the Steklov problem. J. Geom. Anal., 25 (2015), no. 2, 924-950. arXiv:1304.7233.

[Sj] J. Sjöstrand, Singularités analytiques microlocales. Astérisque, 95, 1-166, Soc. Math. France, Paris, 1982.

[Sog] C. D. Sogge, Fourier integrals in classical analysis. Cambridge Tracts in Mathematics, 105, Cambridge University Press, Cambridge, 1993.

[St] M. Stenzel, On the analytic continuation of the Poisson kernel. Monatsh. Math., 178 (2015), no. 2, 299-309.

[T] M. E. Taylor, Partial differential equations II. Qualitative studies of linear equations. Applied Mathematical Sciences, 116. Springer, New York, 2011.

[WZ] X. Wang and J. Zhu, A lower bound for the nodal sets of Steklov eigenfunctions. Math. Res. Lett., 22 (2015), no. 4, 1243-1253. arXiv: 1411.0708.

[Y1] S.-T. Yau, Survey on partial differential equations in differential geometry. Seminar on Differential Geometry, pp. 3-71, Ann. of Math. Stud., 102, Princeton Univ. Press, Princeton, N.J., 1982.

[Y2] S.-T. Yau, Open problems in geometry. Differential geometry: partial differential equations on manifolds (Los Angeles, CA, 1990), 1-28, Proc. Sympos. Pure Math., 54, Part 1, Amer. Math. Soc., Providence, RI, 1993. 
[Z] S. Zelditch, Complex zeros of real ergodic eigenfunctions. Invent. Math., 167 (2007), no. 2, 419-443.

[Z2] S. Zelditch, Pluri-potential theory on Grauert tubes of real analytic Riemannian manifolds, I. Spectral geometry, 299-339, Proc. Sympos. Pure Math., 84, Amer. Math. Soc., Providence, RI, 2012.

[Z3] S. Zelditch, Phase space Weyl laws and eigenfunction growth on Grauert tubes. In preparation.

Department of Mathematics, Northwestern University

EVANSTON, IL 60208-2370, USA

E-mail address: zelditch@math.northwestern.edu

Received September 11, 2014 